\title{
Molecular search with conformational change: One-dimensional discrete-state stochastic model
}

\author{
Jaeoh Shin ${ }^{1}$ and Anatoly B. Kolomeisky ${ }^{1,2,3}$ \\ ${ }^{1}$ Department of Chemistry, Rice University, Houston, Texas 77005, USA \\ ${ }^{2}$ Department of Chemical and Biomolecular Engineering, Rice University, Houston, Texas 77005, USA \\ ${ }^{3}$ Center for Theoretical Biological Physics, Rice University, Houston, Texas 77005, USA
}

(Received 5 August 2018; accepted 7 October 2018; published online 1 November 2018)

\begin{abstract}
Molecular search phenomena are observed in a variety of chemical and biological systems. During the search, the participating particles frequently move in complex inhomogeneous environments with random transitions between different dynamic modes. To understand the mechanisms of molecular search with alternating dynamics, we investigate the search dynamics with stochastic transitions between two conformations in a one-dimensional discrete-state stochastic model. It is explicitly analyzed using the first-passage time probability method to obtain a full dynamic description of the search process. A general dynamic phase diagram is developed. It is found that there are several dynamic regimes in the molecular search with conformational transitions, and they are determined by the relative values of the relevant length scales in the system. Theoretical predictions are fully supported by Monte Carlo computer simulations. Published by AIP Publishing. https://doi.org/10.1063/1.5051035
\end{abstract}

\section{INTRODUCTION}

Many chemical and biological processes involve molecular search that frequently takes place in complex inhomogeneous media, leading to random changes in the dynamic properties of the participating particles. ${ }^{1,2}$ For instance, in the heterogeneous chemical catalysis, the reacting molecules alternate between the fluid phase and the solid surface of the catalyst before the reaction is completed. ${ }^{3}$ Similar alternating dynamics is observed in gene activation by proteins, where the transcription factors associate with specific sequences on DNA. ${ }^{4}$ Experimental studies showed that this process is a combination of three-dimensional (3D) diffusion in the bulk solution and one-dimensional (1D) sliding along the DNA chain. ${ }^{4}$ Such molecular search processes with alternating dynamics are known as intermittent searches, and in recent years, they have been intensively studied both experimentally and theoretically. $1,2,5-22$

The most intriguing feature of the processes with the intermittent search is the random switching of the molecules between different dynamic states. This observation raises several fundamental questions on the mechanisms of such complex processes. Why do natural processes frequently exhibit such complex dynamics? How do these stochastic transitions specifically affect the dynamics? Is the overall dynamics always accelerated by these alternating dynamics? These questions have been discussed before, but the overall molecular picture for the mechanisms of the processes with the intermittent search remains not fully understood. . $^{1,2,15,23}$

One of the simplest intermittent search systems is the 1D case where the reacting molecule searches for the target while

Note: This article is part of the Special Topic "Markov Models of Molecular Kinetics" in J. Chem. Phys. moving along a line and alternating between several conformations with different dynamic properties. This model is relevant for the understanding of how the transcription factors already bound to DNA can locate the specific target sequences. It was shown experimentally that the protein-DNA complexes have different conformations, leading to the variable strength of the protein-DNA interactions during the search process. ${ }^{24-26}$ When the protein molecule weakly interacts with the DNA chain, it can diffuse rapidly, and this is known as a search conformation. But the target can be found only in a so-called recognition conformation when the protein interacts stronger with DNA while diffusing slower. Apparently, alternating between two conformations might help proteins to find the target faster at some conditions. ${ }^{27}$

The molecular search with conformational transitions in 1D systems was theoretically considered before. ${ }^{28-34}$ In Refs. 28-31, the authors presented a continuum model, where the searcher diffuses with alternating diffusivity in the continuum space. However, the analysis was performed only for the search times at one specific location of the target. In addition, in the limit of very small diffusivity in the recognition conformational state, the search times become infinitely large, which is physically unreasonable. This shows that the application of this approach for real biological processes is problematic at these conditions. It has been argued before that a more general discrete-state description is required to properly analyze biochemical search processes. ${ }^{35}$ In Ref. 32, the search dynamics in both continuous and discrete space was analyzed. It was assumed that the molecule can switch between the two regimes: a diffusion conformation from which the target can be found and a so-called teleportation conformation where the searcher can jump to any location in the system, but the target cannot be found. However, the motion in the fast teleportation mode was not considered explicitly, and this also limited the applicability of this model. In Ref. 33, the authors 
considered an infinite lattice of discrete states which are visited by the searching molecules. But the explicit analysis was performed only in the limiting cases with the assumption of conformational equilibrium which restricts the applicability of this approach to real biological systems. Our previous studies of this process investigated 1D conformational changes coupled to the bulk diffusion, and only limiting cases were analyzed. ${ }^{34}$

In this paper, we present a general theoretical approach to describe the molecular search with conformational transitions in one dimension. The searcher molecule stochastically transitions between two dynamic modes with different hopping rates on the lattice, and it can recognize the target only in one of the conformational states. Using a method of firstpassage probabilities, a full dynamic description of the system is obtained, and we concentrate on explicit calculations of the mean search times. By exploring all parameter ranges, several dynamic regimes are identified and described. It is argued that these different regimes are specified by the dominating length scales in the system. Our theoretical analysis is also supported by Monte Carlo computer simulations.

The paper is organized as follows. We introduce the model in Sec. II, and the main results are presented in Sec. III. In Sec. IV, we identify two limiting cases for which physically transparent solutions are obtained. Finally, we summarize and conclude in Sec. V. The details of the calculations are presented in the Appendix.

\section{THEORETICAL MODEL}

We consider the search of a single molecule for a target site in the one-dimensional lattice of discrete sites, as shown in Fig. 1. While the immobile target is located at a single site of the lattice, the searcher molecule diffuses along the lattice. During the process, the molecule can randomly switch between two conformational states: the recognition mode and the search mode. In the recognition mode (labeled as state 1), the molecule can recognize the target site, and it moves with the hopping rate $\mu_{1}$ with equal probability in both directions. In the search mode (labeled as state 2), it cannot recognize the target, but its diffusional hopping rate in this state is $\mu_{2}$, which is generally different from the rate $\mu_{1}$. The stochastic switching between two conformations follows the exponential
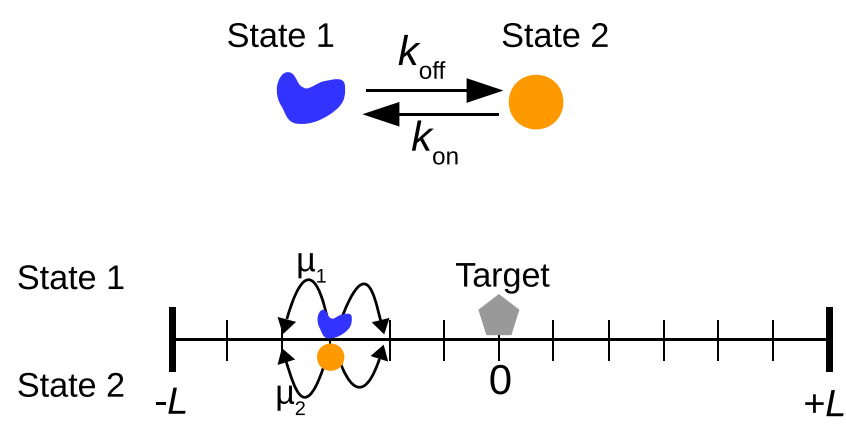

FIG. 1. A schematic view of the molecular search for a target with conformational change. (Top) The molecule switches between two states, and it can recognize the target only when in state 1 . The transition rates between two states are $k_{\text {on }}$ and $k_{\text {off }}$. (Bottom) The hopping rates on the lattice are $\mu_{1}$ and $\mu_{2}$ depending on the state of molecule. waiting-time distributions with the rate $k_{\text {on }}$ (from the search mode to the recognition mode) and $k_{\text {off }}$ (from the recognition mode to the search mode); see Fig. 1. The number of lattice sites is $2 L+1$ which is labeled as $n=-L,-L+1, \ldots, 0, \ldots$, $L-1, L$. We consider reflecting boundary conditions on both sides of the lattice, and for convenience, the target is located at the center $(n=0)$, although our analysis can be extended for any location along the lattice.

To describe the search dynamics in this system, we employ the method of first-passage probabilities that was successful in analyzing various problems related to protein search for targets on DNA. ${ }^{27,34-37}$ We start by defining the first-passage time probability density function $F_{i}(n, t)$ to reach the target at time $t$ given that the molecule was at site $n$ as in the $i$ th state ( $i=1$ or 2 ) at time $t=0$. The temporal evolution of the firstpassage probability functions is governed by the backward master equations, ${ }^{38}$

$$
\begin{aligned}
\frac{\partial F_{1}(n, t)}{\partial t}= & \mu_{1}\left[F_{1}(n-1, t)+F_{1}(n+1, t)\right]+k_{\mathrm{off}} F_{2}(n, t) \\
& -\left(2 \mu_{1}+k_{\mathrm{off}}\right) F_{1}(n, t), \\
\frac{\partial F_{2}(n, t)}{\partial t}= & \mu_{2}\left[F_{2}(n-1, t)+F_{2}(n+1, t)\right]+k_{\mathrm{on}} F_{1}(n, t) \\
& -\left(2 \mu_{2}+k_{\mathrm{on}}\right) F_{2}(n, t),
\end{aligned}
$$

for $-L<n<L$. The physical meaning of these equations is that all trajectories starting on the site $n$ and reaching the target can be divided into three groups: going first to the site $n-1$, going first to the site $n+1$, or switching first to another conformation in the site $n$. At the boundaries $(n= \pm L)$, the equations are slightly different to reflect the geometry of these locations (see the Appendix). The initial condition is $F_{1}(n=0, t)$ $=\delta(t)$, which means that if the initial position of the molecule is at the target site in the conformational state 1 , it finds the target instantaneously.

To solve these equations, we apply the Laplace transform, $\widetilde{F}_{i}(n, s) \equiv \int_{0}^{\infty} F_{i}(n, t) \exp (-s t) d t$, where $s$ is the Laplace variable. Then the above master equations can be rewritten as

$$
\begin{aligned}
\left(s+2 \mu_{1}+k_{\text {off }}\right) \widetilde{F_{1}}(n, s)= & \mu_{1}\left[\widetilde{F_{1}}(n-1, s)+\widetilde{F_{1}}(n+1, s)\right] \\
& +k_{\text {off }} \widetilde{F_{2}}(n, s),
\end{aligned}
$$

$$
\begin{aligned}
\left(s+2 \mu_{2}+k_{\mathrm{on}}\right) \widetilde{F_{2}}(n, s)= & \mu_{2}\left[\widetilde{F_{2}}(n-1, s)+\widetilde{F_{2}}(n+1, s)\right] \\
& +k_{\mathrm{on}} \widetilde{F_{1}}(n, s) .
\end{aligned}
$$

The equations at the boundary are slightly different; see the Appendix. The initial condition in the Laplace domain is now given as $\widetilde{F_{1}}(n=0)=1$. These coupled second-order difference equations can be solved by transforming them into a single fourth-order difference equation. The details of the calculations are presented in the Appendix. Once we obtain $\widetilde{F}_{i}(n, s)$, it is straightforward to compute all dynamic properties in the system. Let us concentrate on the mean search times, or mean-first passage times, which can be obtained from

$$
T_{i}(n)=\int_{0}^{\infty} t F_{i}(n, t) d t=-\left.\frac{\partial \widetilde{F}_{i}(n, s)}{\partial s}\right|_{s=0} .
$$




\section{RESULTS AND DISCUSSION}

Our theoretical method allows us to calculate the mean search times $T_{i}(n)$ for arbitrary kinetic parameters, for any location of the target, and for any lattice size $L$. To be specific, let us put the target in the middle of the lattice $(n=0)$ and assume that at $t=0$ the molecule with equal probability can start from any site in the search conformation $(i=2)$. We will evaluate the position average search time,

$$
T=\left\langle T_{2}(n)\right\rangle=\frac{1}{2 L+1} \sum_{n=-L}^{L} T_{2}(n),
$$

for various sets of kinetic parameters. It is important to note that there are four length scales in the system that are governing the search dynamics. The first one is the size of the lattice $L$, the next one is the size of the target, which takes the single lattice site, and the last two are scanning lengths $\lambda_{1}$ and $\lambda_{2}$, which are defined as $\lambda_{1} \equiv \sqrt{\mu_{1} / k_{\text {off }}}$ and $\lambda_{2} \equiv \sqrt{\mu_{2} / k_{\text {on }}}$. The physical meaning of these scanning lengths is the average distance that the molecule moves along the lattice in one conformational state before switching to the other state.

The results of our calculations for different ranges of kinetic parameters are presented in Figs. 2 and 3. We start with investigating the dependence of the search dynamics on the scanning lengths, and first we consider how the search time $T$ varies as a function of $\lambda_{1}$ in Fig. 2(a). One can identify three different dynamic regimes here. For $\lambda_{1}>L$, the results converge and the mean search times become independent of the hopping rate in the search conformation $\mu_{2}$ and the switching rates between the conformations. This result is easy to understand because for large $\lambda_{1}$ the searching molecule remains mostly in the recognition mode (state 1), and it can find the target without exploring the search conformation (state 2). Another dynamic behavior is observed for $\lambda_{1}<1$. Here the molecule spends most of the time in the search conformation (state 2), with very rare switchings to the recognition mode (state 1). This explains the independence of the mean search time from the scanning length $\lambda_{1}$. Also, in this regime, the target can be reached mostly by the conformational transition from the site $n=0$ in the search mode, and the faster the hopping rate $\mu_{2}$, the higher the probability for this to happen because this site will be visited more frequently. The intermediate dynamic behavior is observed between these two limiting cases, when $1<\lambda_{1}<L$, and the search involves scanning of the lattice in both conformations and frequent changes between them.

Analyzing the dependence of the mean search time on the scanning length $\lambda_{2}$, as shown in Fig. 2(b), three dynamic phases are again observed. For $\lambda_{2}<1$, no dependence on the scanning length is found because the searcher is mostly in state 1 (recognition), and it can find the target without switching to another conformation. In addition, the faster you move in this mode (larger $\mu_{1}$ ), the sooner the target will be located. The dynamics is different for $\lambda_{2}>L$ because the system spends most of the time in the search conformation (state 2) from which the target cannot be located. Decreasing the switching rate $k_{\text {on }}$ (larger $\lambda_{2}$ ) will make finding the target even more difficult. But at the same time, increasing the hopping rate $\mu_{1}$ will accelerate the search because after the rare switch to the recognition mode the target can be found before switching back to the search mode. The dynamic regime when $1<\lambda_{2}<L$ exhibits the intermediate dynamic behavior between these two limiting cases, as expected. In this regime, the mean search time $T$ can be significantly shorter than those of two other regimes, and we argue that it is because the searcher molecule can explore the space fast without much losing its recognition ability of the target. One can also see this from the fact that the most optimal conditions for the search are achieved for the conformational transition rates that is not too slow or too fast.

In a recent paper, ${ }^{39}$ the authors analyzed the hOGG1 protein diffusion on a DNA molecule. By using an optimal estimator of diffusion, they showed that the experimental data are consistent with the diffusion with switching conformation with different diffusivity. It is estimated that the protein spends a similar time in the fast search mode and slow recognition mode, and the diffusion constant for the former is about three to ten times faster than that of the latter. In our language, $k_{\text {on }} \simeq k_{\text {off }}$ and $\mu_{2} \simeq(3-10) \mu_{1}$. Intriguingly, in our model, for the case of $\mu_{2}=10 \mu_{1}$, the search time is minimum when $k_{\text {on }} \simeq k_{\text {off }}$, indicating that the protein moves with the optimal parameters that can minimize the search time; see Fig. 2(b), blue line. This also opens a question if other DNA-binding proteins with conformational transitions search for their target with the optimal kinetic parameters.

It is interesting to note that the conformational transitions dominate in the intermediate dynamic regimes $\left(1<\lambda_{1}\right.$,

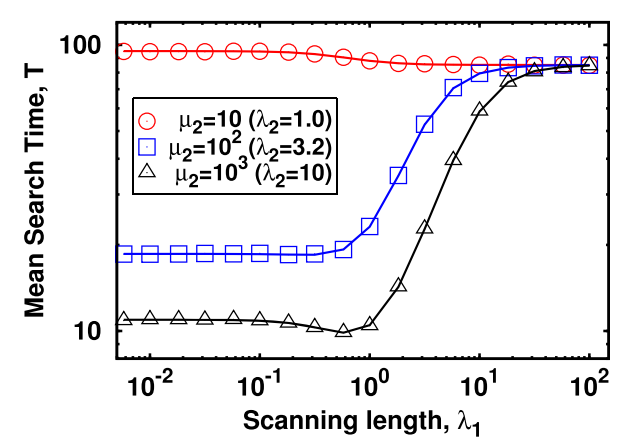

(a)

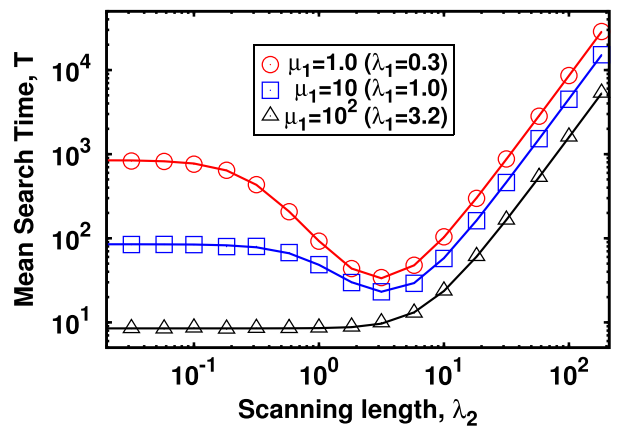

(b)
FIG. 2. The position-averaged mean search time $T$ as a function of the scanning lengths $\lambda_{1}$ and $\lambda_{2}$. (a) Here $k_{\mathrm{on}}=\mu_{1}=10$ and three different values of $\mu_{2}$ are utilized. We varied $k_{\text {off }}$ to change $\lambda_{1}$. (b) Here $\mu_{2}=100, k_{\text {off }}$ $=10$, and three different values of $\mu_{1}$ are utilized. We varied $k_{\text {on }}$ to change $\lambda_{2}$. The solid lines are from the exact formulas, and the symbols are from the Monte Carlo computer simulations. The lattice size is $2 L+1=101$, and the target is at the center of the lattice. 


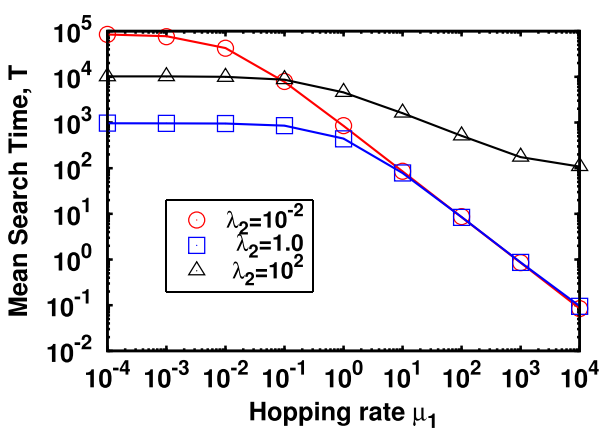

(a)

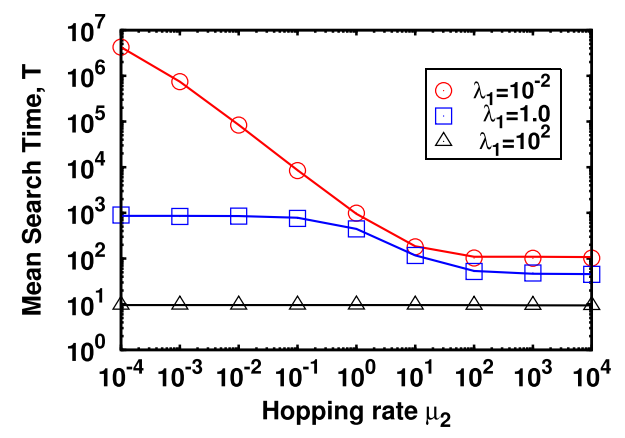

(b)
FIG. 3. The position-averaged mean search times $T$ as a function of the hopping rates in different conformations. (a) Here $\mu_{2}=100$ and $k_{\text {off }}=1$ and three values of $k_{\text {on }}$ are utilized. (b) Here $\mu_{1}=100$ and $k_{\mathrm{on}}=1$ and three values of $k_{\text {off }}$ are utilized. The solid lines are from the exact formulas, and the symbols are from the Monte Carlo computer simulations. The lattice size is $2 L+1=101$, and the target is at the center of the lattice.
$\lambda_{2}<L$ ), but this does not always lead to the most optimal search dynamics: see the two upper curves in Fig. 2(a) and the lower curve in Fig. 2(b). It can only happen if switching helps explore the phase space more rapidly. This suggests that in the chemical and biological systems with the alternating dynamics there is an optimal range of the transition rates that can accelerate the search dynamics. Thus, in contrast to some widely expressed views, it is generally not valid to claim that just engaging in the stochastic switching will always accelerate the search dynamics.

To explore more the dynamic properties of the system with stochastic switchings between conformations, we calculate the dependence of the search times on the hopping rates $\mu_{1}$ and $\mu_{2}$, and the results are presented in Fig. 3. One can see that for relatively small values of $\mu_{1}$ (hopping rate in the recognition mode) the mean search times do not depend on them because in this case the system is mostly found in the search mode and the target can be reached via the stochastic transition at $n=$ 0 [Fig. 3(a)]. Only for larger values of $\mu_{1}$, the target can also be reached from sliding along the lattice in the recognition mode, and increasing $\mu_{1}$ lowers the search time. A different behavior is observed for the dependence of $T$ as a function of

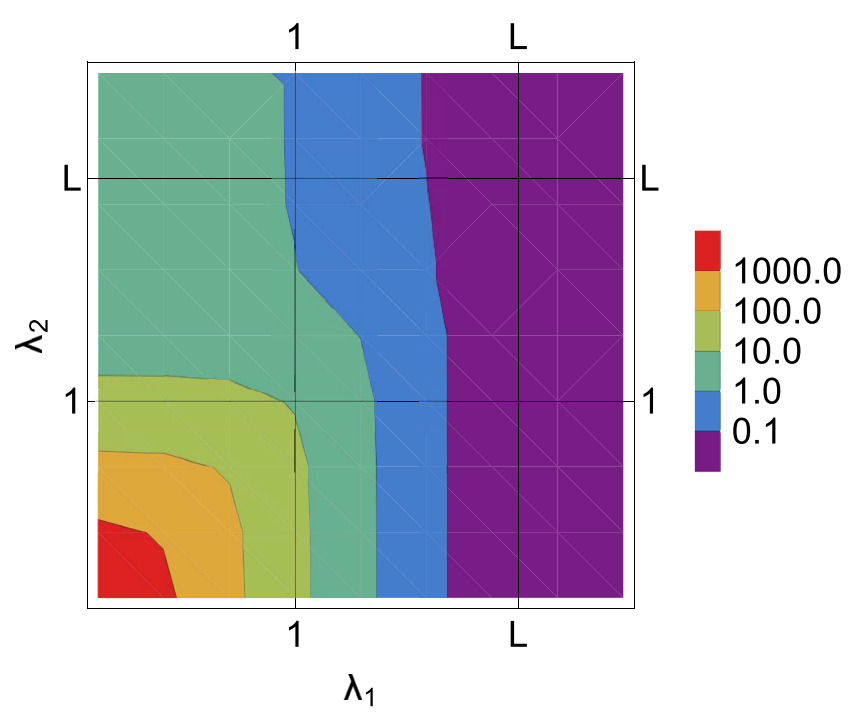

FIG. 4. Dynamic phase diagram for the molecular search with stochastic conformational transitions. The calculations used the lattice size $2 L+1=101$, $k_{\text {on }}=k_{\text {off }}=100$, and we varied $\mu_{1}$ and $\mu_{2}$. The color map shows the values of the position averaged mean search times $T$. Lines schematically separate different dynamic regimes. $\mu_{2}$; see Fig. 3(b). Increasing the hopping rate $\mu_{2}$ (the hopping rate in the search mode) accelerates the search because the system can explore faster the overall space, while for large $\mu_{2}$, the search dynamics becomes independent of the hopping rate. In this case, other processes (stochastic transition from the search mode to the recognition mode at the site $n=0$ ) are the rate-limiting step in the search process. It is also clear that $T$ is independent of $\mu_{2}$ for large values of $\lambda_{1}$; see the lower curve in Fig. 3(b). This corresponds to the situation when the molecule is almost always in the recognition mode, and the target can be found without going into another conformational state.

Our theoretical analysis fully agrees with Monte Carlo computer simulations, and it suggests that the dynamic behavior in the system is determined by the relative values of the several length scales such as the scanning length $\lambda_{1}, \lambda_{2}$, the size of the system $L$, and the size of the target site (taken to be equal to unity). Combining these considerations together, we develop a general dynamic phase diagram for the molecular search with stochastic conformational transitions. The results are presented in Fig. 4. One can see that up to nine dynamic phases can be identified, showing a very rich behavior even in the relatively simple system with conformational transitions between only two modes.

\section{LIMITING CASES}

Although our theoretical method provides explicit solutions for all ranges of kinetic parameters, to explain better the molecular search with alternating dynamics, it is convenient to consider some limiting cases. In these situations, more transparent analysis can be performed, which might clarify better the mechanisms of the search processes with stochastic transitions. There are two cases, corresponding to $\lambda_{1} \gg \lambda_{2}$ and $\lambda_{2} \gg \lambda_{1}$, which can be treated this way.

\section{A. $\lambda_{1} \gg \lambda_{2}$ case}

In this regime, the molecule spends most of the time in the recognition mode with occasional rare transitions to the search mode, in which it does not slide and switches back. Then the dynamics can be well described by assuming $\mu_{2}=0$. Using this result in Eq. (2) leads to

$$
\frac{\partial F_{2}(n, t)}{\partial t}=k_{\mathrm{on}} F_{1}(n, t)-k_{\mathrm{on}} F_{2}(n, t),
$$


which in the Laplace domain can be written as

$$
\left(s+k_{\mathrm{on}}\right) \widetilde{F_{2}}(n, s)=k_{\mathrm{on}} \widetilde{F_{1}}(n, s) .
$$

Now analyzing Eqs. (3) and (4) for $\mu_{2}=0$ corresponds to solving a second-order difference equation instead of the much more complex fourth-order difference equation for the general situation. A similar analysis has been performed in previous studies on the protein search for targets on DNA. $27,34,35$

We assume that the general solution is of the form $\widetilde{F_{1}}(n) \simeq A x^{n}$, which leads to a quadratic equation

$$
\begin{aligned}
& \mu_{1}\left(s+k_{\mathrm{on}}\right) x^{2}-\left[s^{2}+s\left(2 \mu_{1}+k_{\mathrm{on}}+k_{\mathrm{off}}\right)+2 \mu_{1} k_{\mathrm{on}}\right] x \\
& \quad+\mu_{1}\left(s+k_{\mathrm{on}}\right)=0,
\end{aligned}
$$

with roots given by

$$
x_{1}=\frac{s^{2}+s\left(2 \mu_{1}+k_{\text {on }}+k_{\text {off }}\right)+2 \mu_{1} k_{\text {on }}-\sqrt{D}}{2 \mu_{1}\left(s+k_{\text {on }}\right)}, \quad x_{2}=1 / x_{1},
$$

while the parameter $D$ is equal to

$D=\left[s^{2}+s\left(2 \mu_{1}+k_{\text {on }}+k_{\text {off }}\right)+2 \mu_{1} k_{\text {on }}\right]^{2}-4 \mu_{1}^{2}\left(s+k_{\text {on }}\right)^{2}$.

Then the general solution is $\widetilde{F_{1}}(n)=A_{1} x_{1}^{n}+A_{2} x_{1}^{-n}$, where $x_{1}$ is given by Eq. (10) and $A_{1}$ and $A_{2}$ are the unknown coefficients that can be determined from the boundary conditions. The final expression for the first-passage probability function (in the Laplace form) is

$$
\widetilde{F_{1}}(n)=\frac{x_{1}^{2 L+n}}{x_{1}^{2 L}+1}+\frac{x_{1}^{-n}}{x_{1}^{2 L}+1} .
$$

This yields the following expression for the mean search time from the site $n$ in the recognition conformation,

$$
T_{1}(n)=\frac{|n|(2 L+1-|n|)}{2 \mu_{\mathrm{eff} 1}},
$$

where $\mu_{\text {eff } 1}=\mu_{1} /\left(1+k_{\text {off }} / k_{\text {on }}\right)$. This result has a simple physical interpretation. It describes the mean first-passage time of finding the target (which is at the origin) starting from the site $n$ by purely $1 \mathrm{D}$ motion in the segment of size $(2 L+1)$ with the effective hopping rate $\mu_{\text {eff } 1}$. In this regime, the molecule explores the lattice mostly in the search conformation with the occasional switchings to the immobile recognition mode. At large times, the system reaches the effective equilibrium between two conformations because the particle does not slide in the recognition mode. Therefore the hopping rate is rescaled by an equilibrium fraction of finding the molecule in the search configuration, $f=\frac{k_{\mathrm{on}}}{k_{\mathrm{on}}+k_{\mathrm{off}}}$.

Finally, it can be shown that the position-average mean search time is

$$
T=\frac{1}{k_{\mathrm{on}}}+\frac{1}{2 L+1} \frac{1}{\mu_{\mathrm{eff} 1}} \sum_{n=1}^{L} n(2 L+1-n) .
$$

The first term corresponds to the transition time from state 2 to state 1 , and the second term corresponds to 1D search time with effective hopping rate $\mu_{\text {eff1 }}$. It can be shown that these results fully agree with exact calculations in the limit of very small $\lambda_{2}$.

\section{B. $\lambda_{1} \ll \lambda_{2}$ case}

Exact calculations can also be performed in the opposite limit when the searching molecule moves along the lattice mostly in the search mode with occasional switchings to the recognition mode. In this regime, the target is reached only via the conformational transitions from the site $n=0$, and we can approximate the dynamics as $\mu_{1}=0$, modifying Eq. (1) as

$$
\frac{\partial F_{1}(n, t)}{\partial t}=k_{\mathrm{off}} F_{2}(n, t)-k_{\mathrm{off}} F_{1}(n, t) .
$$

Again using the Laplace transformations, the mean search time $T$ can be explicitly evaluated. The final expression is

$$
T_{2}(n)=\frac{1}{2 \mu_{\mathrm{eff} 2}}|n|(2 L-|n|+1)+\left[\frac{2 L+1}{k_{\mathrm{on}}}+\frac{2 L}{k_{\mathrm{off}}}\right],
$$

where $\mu_{\text {eff } 2}=\mu_{2} /\left(1+k_{\text {on }} / k_{\text {off }}\right)$. Here the first term describes the mean time to travel from the initial position $n$ to the site $n=0$ in the search conformation, and the second term corresponds to the time of conformational transition from state 2 to state 1 and return back to the search conformation. The number of transitions from state 2 to state 1 is $2 L+1$, while the number of reversed transitions is less by one because the last transition to the recognition mode is going to be directly to the target, which ends the search process. It can be shown explicitly that these results agree with the full solutions in this limit.

\section{SUMMARY AND CONCLUSIONS}

We presented a comprehensive theoretical analysis of the one-dimensional discrete-state stochastic model of the molecular search with random conformational transitions. Stimulated by biological processes of protein-DNA interactions, this model also serves as a general testing ground for understanding the role of the intermittent search phenomena in complex natural systems. Using the method of first-passage probabilities, we were able to obtain the explicit quantitative description of the dynamics for all ranges of kinetic parameters. It has been argued that four length scales specify the dynamic phase diagram in this system. They include the size of the target site, the size of the system, and the two scanning lengths in different conformations. A general dynamic phase diagram that describes all possible search behaviors is constructed. It is found that the stochastic transitions between two conformations might optimize the search dynamics at the conditions when the switching leads to a more rapid exploration of the phase space without too much losing the recognition ability. Our theoretical calculations also show that the acceleration does not always happen, indicating that the intermittent search is not always the most efficient dynamic regime. In addition, because our model is one-dimensional, any changes in the dynamic properties cannot be associated with the "lowering of dimensionality" arguments that are widely expressed in the literature for the intermittent search phenomena. ${ }^{5}$

The presented theoretical model gives a clear picture of how the stochastic switching is affecting the search dynamics and clarifies many aspects of the intermittent search process. 
However, it is important to note that this model needs to be improved to make it more realistic. In particular, one needs to include the reversible desorption and rebinding events to make it more applicable to real biological phenomena. ${ }^{30,31,34,39}$ It will be interesting to test these theoretical predictions in experimental systems as well as in the more advanced theoretical descriptions. While the motivation of our model is the single protein search for a target, it can be extended to the case of multiple interacting search molecules, which can help explain recent studies on the dynamic clustering of activating transcription factors. ${ }^{40}$

\section{ACKNOWLEDGMENTS}

The work was supported by the Welch Foundation (C-1559), by the NSF (Award No. CHE-1664218), and by the Center for Theoretical Biological Physics sponsored by the NSF (Award No. PHY-1427654).

\section{APPENDIX: DETAILS OF CALCULATIONS}

Here we describe the details of the first-passage probability calculations to evaluate the search dynamics. We define the first-passage time probability density to find the target with the initial position $n$ at time $t=0$ in state $i(=1$ or 2$)$ as $F_{i}(n, t)$. The number of lattice sites is $2 L+1$, labeled as $-L,-L+1, \ldots$, $0, \ldots, L-1, L$. We consider the target at the center of the lattice, $n=0$. The evolution of $F_{i}(n, t)$ follows the following backward master equations: ${ }^{34,35}$

$$
\begin{aligned}
\frac{\partial F_{1}(n, t)}{\partial t}= & \mu_{1}\left[F_{1}(n-1, t)+F_{1}(n+1, t)\right]+k_{\mathrm{off}} F_{2}(n, t) \\
& -\left(2 \mu_{1}+k_{\mathrm{off}}\right) F_{1}(n, t), \\
\frac{\partial F_{2}(n, t)}{\partial t}= & \mu_{2}\left[F_{2}(n-1, t)+F_{2}(n+1, t)\right]+k_{\mathrm{on}} F_{1}(n, t) \\
& -\left(2 \mu_{2}+k_{\mathrm{on}}\right) F_{2}(n, t),
\end{aligned}
$$

for $-L<n<+L$. At the boundary $n=-L$, we have

$$
\begin{aligned}
\frac{\partial F_{1}(-L, t)}{\partial t}= & \mu_{1} F_{1}(-L+1, t)+k_{\mathrm{off}} F_{2}(-L, t) \\
& -\left(\mu_{1}+k_{\mathrm{off}}\right) F_{1}(-L, t), \\
\frac{\partial F_{2}(-L, t)}{\partial t}= & \mu_{2} F_{2}(-L+1, t)+k_{\mathrm{on}} F_{1}(-L, t) \\
& -\left(\mu_{2}+k_{\mathrm{on}}\right) F_{2}(-L, t) .
\end{aligned}
$$

Similar equations can be written for $n=L$.

Now the Laplace transform, $\widetilde{F}_{i}(n, s) \equiv \int_{0}^{\infty} F_{i}(n, t)$ $\exp (-s t) d t$, where $s$ is the Laplace variable, can be applied for all master equations to transform them from the differential equations into algebraic equations. This leads to

$\left(s+2 \mu_{1}+k_{\text {off }}\right) \widetilde{F_{1}}(n)=\mu_{1}\left[\widetilde{F_{1}}(n-1)+\widetilde{F_{1}}(n+1)\right]+k_{\text {off }} \widetilde{F_{2}}(n)$,

$\left(s+2 \mu_{2}+k_{\text {on }}\right) \widetilde{F_{2}}(n)=\mu_{2}\left[\widetilde{F_{2}}(n-1)+\widetilde{F_{2}}(n+1)\right]+k_{\text {on }} \widetilde{F_{1}}(n)$
These expressions form a system of two coupled second-order difference equations. To solve this system, these two equations can be combined to make them a single fourth-order difference equation. This procedure yields

$$
\widetilde{F_{1}}(n)=\frac{\left(s+2 \mu_{2}+k_{\mathrm{on}}\right)}{k_{\mathrm{on}}} \widetilde{F_{2}}(n)-\frac{\mu_{2}}{k_{\mathrm{on}}}\left[\widetilde{F_{2}}(n-1)+\widetilde{F_{2}}(n+1)\right] .
$$

Substituting this into Eq. (A5) yields

$$
\begin{aligned}
\left(a_{1} a_{2}+\right. & \left.2 \mu_{1} \mu_{2}-k_{\text {on }} k_{\text {off }}\right) \widetilde{F_{2}}(n) \\
= & \left(a_{1} \mu_{2}+a_{2} \mu_{1}\right)\left[\widetilde{F_{2}}(n-1)+\widetilde{F_{2}}(n+1)\right] \\
& -\mu_{1} \mu_{2}\left[\widetilde{F_{2}}(n-2)+\widetilde{F_{2}}(n+2)\right],
\end{aligned}
$$

where new auxiliary functions are defined as $a_{1} \equiv s+2 \mu_{1}+k_{\text {off }}$ and $a_{2} \equiv s+2 \mu_{2}+k_{\text {on }}$. Now, introducing $A \equiv a_{1} a_{2}+2 \mu_{1} \mu_{2}$ $-k_{\text {on }} k_{\text {off }}, B \equiv a_{1} \mu_{2}+a_{2} \mu_{1}$, and $C \equiv \mu_{1} \mu_{2}$, we can rewrite Eq. (A8) in the more compact form

$$
\begin{aligned}
A \widetilde{F_{2}}(n)= & B\left[\widetilde{F_{2}}(n-1)+\widetilde{F_{2}}(n+1)\right] \\
& -C\left[\widetilde{F_{2}}(n-2)+\widetilde{F_{2}}(n+2)\right] .
\end{aligned}
$$

To solve this equation, we assume that the solution is of the form $\widetilde{F_{2}}(n)=\alpha x^{n}$, and substituting this into Eq. (A9) gives a quartic equation,

$$
C x^{4}-B x^{3}+A x^{2}-B x+C=0 .
$$

It can be shown that it has four roots of the form $\left(x_{1}, 1 / x_{1}, x_{2}\right.$, $\left.1 / x_{2}\right)$, and $x_{i}(i=1$ or 2$)$ might be a complex number. Then the general solution is $\widetilde{F_{2}}(n)=A_{1} x_{1}^{n}+A_{2} x_{1}^{-n}+B_{1} x_{2}^{n}+B_{2} x_{2}^{-n}$. We need to determine the four unknown coefficients $\left(A_{1}, A_{2}, B_{1}\right.$, and $B_{2}$ ) by using the following boundary conditions:

(1) At $n=-L$, the corresponding master equations in the Laplace domain are

$$
\begin{aligned}
& {\left[s+\mu_{1}+k_{\mathrm{off}}\right] \widetilde{F_{1}}(-L)=\mu_{1} \widetilde{F_{1}}(-L+1)+k_{\mathrm{off}} \widetilde{F_{2}}(-L),} \\
& {\left[s+\mu_{2}+k_{\mathrm{on}}\right] \widetilde{F_{2}}(-L)=\mu_{2} \widetilde{F_{2}}(-L+1)+k_{\mathrm{on}} \widetilde{F_{1}}(-L) .}
\end{aligned}
$$

Substituting Eq. (A12) into Eq. (A11) gives

$$
\begin{aligned}
{\left[s+\mu_{1}\right.} & \left.+k_{\mathrm{off}}\right]\left[\left(s+\mu_{2}+k_{\mathrm{on}}\right) \widetilde{F_{2}}(-L)-\mu_{2} \widetilde{F_{2}}(-L+1)\right] \\
= & \mu_{1}\left[\left(s+2 \mu_{2}+k_{\mathrm{on}}\right) \widetilde{F_{2}}(-L+1)-\mu_{1}\left(\widetilde{F_{2}}(-L)\right.\right. \\
& \left.\left.+\widetilde{F_{2}}(-L+2)\right)\right]+k_{\mathrm{on}} k_{\mathrm{off}} \widetilde{F_{2}}(-L) .
\end{aligned}
$$

This can be written as

$$
\begin{aligned}
{\left[\left(s+\mu_{1}+k_{\mathrm{off}}\right)\left(s+\mu_{2}+k_{\mathrm{on}}\right)+\mu_{1} \mu_{2}-k_{\mathrm{on}} k_{\mathrm{off}}\right] \widetilde{F_{2}}(-L) } \\
\left.=\left[\left(s+\mu_{1}+k_{\mathrm{off}}\right) \mu_{2}+\mu_{1} a_{2}\right)\right] \widetilde{F_{2}}(-L+1) \\
\quad-\mu_{1} \mu_{2} \widetilde{F_{2}}(-L+2) .
\end{aligned}
$$

This is the first boundary condition.

(2) The master equations for the site $n=-L+1$ in two conformational states are

$$
a_{1} \widetilde{F_{1}}(-L+1)=\mu_{1}\left[\widetilde{F_{1}}(-L)+\widetilde{F_{1}}(-L+2)\right]+k_{\text {off }} \widetilde{F_{2}}(-L+1)
$$

$a_{2} \widetilde{F_{2}}(-L+1)=\mu_{2}\left[\widetilde{F_{2}}(-L)+\widetilde{F_{2}}(-L+2)\right]+k_{\text {on }} \widetilde{F_{1}}(-L+1)$ 
We can also rewrite the master equations for the sites $n=-L$, $n=-L+1$, and $n=-L+2$,

$k_{\mathrm{on}} \widetilde{F_{1}}(-L+1)=a_{2} \widetilde{F_{2}}(-L+1)-\mu_{2}\left[\widetilde{F_{2}}(-L)+\widetilde{F_{2}}(-L+2)\right]$,

$k_{\mathrm{on}} \widetilde{F_{1}}(-L)=\left(s+\mu_{2}+k_{\mathrm{on}}\right) \widetilde{F_{2}}(-L)-\mu_{2} \widetilde{F_{2}}(-L+1)$,

$k_{\mathrm{on}} \widetilde{F_{1}}(-L+2)=a_{2} \widetilde{F_{2}}(-L+2)-\mu_{2}\left[\widetilde{F_{2}}(-L+1)+\widetilde{F_{2}}(-L+3)\right]$.

Substituting them into Eq. (A15) yields

$$
\begin{aligned}
a_{1}\left[a_{2} \widetilde{F_{2}}\right. & \left.(-L+1)-\mu_{2}\left\{\widetilde{F_{2}}(-L)+\widetilde{F_{2}}(-L+2)\right\}\right] \\
= & \mu_{1}\left[\left(s+\mu_{2}+k_{\mathrm{on}}\right) \widetilde{F_{2}}(-L)-\mu_{2} \widetilde{F_{2}}(-L+1)\right. \\
& \left.+a_{2} \widetilde{F_{2}}(-L+2)-\mu_{2}\left\{\widetilde{F_{2}}(-L+1)+\widetilde{F_{2}}(-L+3)\right\}\right] \\
& +k_{\mathrm{on}} k_{\mathrm{off}} \widetilde{F_{2}}(-L+1),
\end{aligned}
$$

which can be simplified as

$$
\begin{aligned}
& {\left[a_{1} \mu_{2}+\left(s+\mu_{2}+k_{\mathrm{on}}\right) \mu_{1}\right] \widetilde{F_{2}}(-L)-\left[a_{1} a_{2}+2 \mu_{1} \mu_{2}-k_{\mathrm{on}} k_{\mathrm{off}}\right]} \\
& \quad \times \widetilde{F_{2}}(-L+1)+\left[a_{1} \mu_{2}+a_{2} \mu_{1}\right] \widetilde{F_{2}}(-L+2) \\
& \quad-\mu_{1} \mu_{2} \widetilde{F_{2}}(-L+3)=0 .
\end{aligned}
$$

This is the second boundary condition.

(3) The additional boundary condition is associated with the dynamics at the target site because $\widetilde{F}_{1}(n=0)=1$. Equation (A6) with $n=0$ is

$$
a_{2} \widetilde{F_{2}}(0)=\mu_{2}\left(\widetilde{F_{2}}(-1)+\widetilde{F_{2}}(1)\right)+k_{\text {on }} .
$$

Because of the symmetry of the system, $\widetilde{F_{2}}(-1)=\widetilde{F_{2}}(1)$ so that the above equation simplifies into

$$
a_{2} \widetilde{F_{2}}(0)=2 \mu_{2} \widetilde{F_{2}}(-1)+k_{\text {on }} .
$$

This is the third boundary condition.

(4) From master equations for $n=-1$ and $n=-2$, one can derive

$$
\begin{aligned}
& k_{\mathrm{on}} \widetilde{F_{1}}(-1)=a_{2} \widetilde{F_{2}}(-1)-\mu_{2}\left\{\widetilde{F_{2}}(-2)+\widetilde{F_{2}}(0)\right\}, \\
& k_{\mathrm{on}} \widetilde{F_{1}}(-2)=a_{2} \widetilde{F_{2}}(-2)-\mu_{2}\left\{\widetilde{F_{2}}(-3)+\widetilde{F_{2}}(-1)\right\} .
\end{aligned}
$$

From Eq. (A5) with $n=-1$, we have

$$
a_{1} \widetilde{F_{1}}(-1)=\mu_{1}\left\{\widetilde{F_{1}}(-2)+\widetilde{F_{1}}(0)\right\}+k_{\text {off }} \widetilde{F_{2}}(-1) .
$$

Substituting Eq. (A22) into Eq. (A23) yields

$$
\begin{aligned}
a_{1}\left[a_{2} \widetilde{F_{2}}(-1)-\mu_{2}\left\{\widetilde{F_{2}}(-2)+\widetilde{F_{2}}(0)\right\}\right] \\
=\mu_{1}\left[a_{2} \widetilde{F_{2}}(-2)-\mu_{2}\left\{\widetilde{F_{2}}(-3)+\widetilde{F_{2}}(-1)\right\}\right] \\
\quad+\mu_{1} k_{\mathrm{on}}+k_{\mathrm{on}} k_{\mathrm{off}} \widetilde{F_{2}}(-1),
\end{aligned}
$$

which can be further simplified as

$$
\begin{aligned}
\mu_{1} \mu_{2} \widetilde{F_{2}}(-3)-\left(a_{1} \mu_{2}+a_{2} \mu_{1}\right) \widetilde{F_{2}}(-2)+\left(a_{1} a_{2}+\mu_{1} \mu_{2}-k_{\text {on }} k_{\text {off }}\right) \\
\quad \times \widetilde{F_{2}}(-1)-a_{1} \mu_{2} \widetilde{F_{2}}(0)=\mu_{1} k_{\text {on }} .
\end{aligned}
$$

This is the fourth boundary condition.
These boundary conditions are considered together, and numerical solutions are obtained for the unknown coefficients $A_{1}, A_{2}, B_{1}$, and $B_{2}$. Then Eq. (5) is utilized for explicit calculations of the mean search times.

${ }^{1}$ O. Bénichou, C. Loverdo, M. Moreau, and R. Voituriez, Rev. Mod. Phys. 83(1), 81 (2011).

${ }^{2}$ P. C. Bressloff and J. M. Newby, Rev. Mod. Phys. 85, 135 (2013).

${ }^{3}$ J. R. H. Ross, Heterogeneous Catalysis: Fundamentals and Applications (Elsevier, Amsterdam, 2011).

${ }^{4}$ J. Gorman and E. C. Greene, Nat. Struct. Mol. Biol. 15, 768 (2008).

${ }^{5}$ G. Adam and M. Delbruck, in Structural Chemistry and Molecular Biology, edited by A. Rich and N. Davidson (Freeman, San Francisco, 1968).

${ }^{6}$ A. D. Riggs, S. Bourgeois, and M. Cohn, J. Mol. Biol. 53(3), 401 (1970).

${ }^{7}$ O. G. Berg and C. Blomberg, Biophys. Chem. 4(4), 367 (1976).

${ }^{8}$ O. G. Berg, R. B. Winter, and P. H. Von Hippel, Biochemistry 20, 6929 (1981).

${ }^{9}$ P. H. von Hippel and O. G. Berg, J. Biol. Chem. 264, 675 (1989).

${ }^{10}$ M. Coppey, O. Bénichou, R. Voituriez, and M. Moreau, Biophys. J. 87, 1640 (2004)

${ }^{11}$ M. Slutsky and L. A. Mirny, Biophys. J. 87, 4021 (2004).

${ }^{12}$ A. G. Cherstvy, A. B. Kolomeisky, and A. A. Kornyshev, J. Phys. Chem. B 112(15), 4741 (2008).

${ }^{13}$ M. A. Lomholt, B. van den Broek, S.-M. J. Kalisch, G. J. L. Wuite, and R. Metzler, Proc. Natl. Acad. Sci. U. S. A. 106, 8204 (2009).

${ }^{14}$ L. Mirny, M. Slutsky, Z. Wunderlich, A. Tafvizi, J. Leith, and A. Kosmrlj, J. Phys. A: Math. Theor. 42(43), 434013 (2009).

${ }^{15}$ A. B. Kolomeisky, Phys. Chem. Chem. Phys. 13, 2088 (2011).

${ }^{16}$ E. F. Koslover, M. A. D. de la Rosa, and A. J. Spakowitz, Biophys. J. 101, 856 (2011).

${ }^{17}$ M. Bauer and R. Metzler, Biophys. J. 102, 2321 (2012).

${ }^{18}$ M. Bauer and R. Metzler, PLoS One 8, e53956 (2013).

${ }^{19}$ M. Bauer, E. S. Rasmussen, M. A. Lomholt, and R. Metzler, Sci. Rep. 5, 10072 (2015).

${ }^{20}$ L. Liu, A. G. Cherstvy, and R. Metzler, J. Phys. Chem. B 121(6), 1284 (2017).

${ }^{21}$ A. Godec and R. Metzler, J. Phys. A: Math. Theor. 50, 084001 (2017).

${ }^{22}$ P. Kar, A. G. Cherstvy, and R. Metzler, Phys. Chem. Chem. Phys. 20, 7931 (2018).

${ }^{23}$ M. Sheinman, O. Bénichou, Y. Kafri, and R. Voituriez, Rep. Prog. Phys. 75, 026601 (2012).

${ }^{24}$ C. G. Kalodimos et al., Science 305, 386 (2004).

${ }^{25}$ A. Tafvizi, F. Huang, A. R. Fersht, L. A. Mirny, and A. M. van Oijen, Proc. Natl. Acad. Sci. U. S. A. 108, 563 (2011).

${ }^{26}$ J. Iwahara and Y. Levy, Transcription 4, 58 (2013).

${ }^{27}$ M. P. Kochugaeva, A. A. Berezhkovskii, and A. B. Kolomeisky, J. Phys. Chem. Lett. 8, 4049 (2017).

${ }^{28}$ J. Reingruber and D. Holcman, Phys. Rev. Lett. 103, 148102 (2009).

${ }^{29}$ J. Reingruber and D. Holcman, J. Phys.: Condens. Matter 22, 065103 (2010).

${ }^{30}$ J. Reingruber and D. Holcman, Phys. Rev. E 84, 020901 (2011).

${ }^{31}$ J. Cartailler and J. Reingruber, Phys. Biol. 12, 046012 (2015).

${ }^{32}$ O. Bénichou, M. Moreau, P.-H. Sueta, and R. Voituriez, J. Chem. Phys. 126, 234109 (2007).

${ }^{33}$ K. L. Ivanov and N. N Lukzen, Chem. Phys. 435, 14 (2014).

${ }^{34}$ M. P. Kochugaeva, A. A. Shvets, and A. B. Kolomeisky, J. Phys. A: Math. Theor. 49, 444004 (2016).

${ }^{35}$ A. Veksler and A. B. Kolomeisky, J. Phys. Chem. B 117(42), 12695 (2013).

${ }^{36}$ A. A. Shvets and A. B. Kolomeisky, J. Chem. Phys. 143, 245101 (2015).

${ }^{37}$ J. Shin and A. B. Kolomeisky, J. Phys. Chem. B 122, 2243 (2018).

${ }^{38}$ S. Redner, A Guide to First-Passage Processes (Cambridge University Press, Cambridge, UK, 2001).

${ }^{39}$ C. L. Vestergaard, P. C. Blainey, and H. Flyvbjerg, Nucleic Acids Res. 46, 2446 (2018).

${ }^{40}$ A. J. Plys and R. E. Kingston, Science 361, 329 (2018). 